established by Davis in 1974, called the classical comparison method. Subsequently, other methods have appeared, such as the minimum effective dose method, the dose response method, the consensus among experts such as consensus method by Gardner, and the Daily Dose method of the World Health Organization. In 2016, Leucht et al performed the meta-analysis comparing the equivalence by the alternative methods of secondgeneration antipsychotics orally, based on Olanzapine. However, therapeutic equivalences between injectable antipsychotics have not yet been made.

The objective of the study is to establish a pattern of therapeutic equivalences between long-acting antipsychotics, based on the method of the Defined Daily Dose (DDD).

METHOD: The DDD is the dose of the maintenance medium of a drug for its main indication in adults of $70 \mathrm{~kg}$. In the case of antipsychotics, psychosis is the most important indication. DDDs are different for each route of administration, especially if the bioavailability of the drug varies between one route and another. To establish the DDD of a drug, 3 measures are taken: firstly, the dose ranges of the drug approved by at least 1 major regulatory authority. Secondly, doses used in clinical trials. Thirdly, post marketing data on dose used in clinical practice when the drug is commercialized. Depot formulations are usually assigned the same DDDs as the ordinary oral dosage form. Based on the DDD according to the WHO classification at http://www.whocc.no/. For comparison, Olanzapine $210 \mathrm{mg}$ was used as the main drug and equivalences were established from it. Therapeutic deposit of Aripiprazole (ARI), Flufenazine decanoate (FLU), Haloperidol Decanoate (HAL) Olanzapine pamoate (OLA), Paliperidone palmitate (PAL), Risperidone depot (RIS), and Zuclopenthixol decanoate (ZUC).

RESULTS: The results will be shown in a $8 x 8$ table.

CONCLUSIONS: DDD is available for almost all antipsychotics and is an accepted method as well as a clinical level as a researcher. They are based on a wide variety of data from different sources. Several studies have found a strong correlation between this method and other methods of equivalence. This method also has limitations. First, the DDDs were not established for the purpose of therapeutic equivalences. Secondly, the daily dose can be applied mainly to the efficacy of the drug, when the dose could cause some adverse effects.

The establishment of therapeutic equivalences may help when a clinician needs to change one long-term antipsychotic. This could reduce psychotic relapses. It may enhance therapeutic adherence avoiding undesirable side effects. On the other hand, long-acting antipsychotics have corroborated the adherence and decrease of relapses, which is why it is increasingly used as a good alternative to oral drugs.

12

\section{Impact of Aripiprazole Long-acting Injectable (ALAI) Initiation on Hospitalizations and Visits to Emergency}

Carlos Parro-Torres, $M D^{\prime}$; Elena Ros-Cucurull, $M D^{2}$; and Sergio Arques-Egea, $M^{3}$

${ }^{1}$ Gregorio Marañon University General Hospital, Madrid, Spain

${ }^{2}$ Vall d'Hebron Hospital, Barcelona, Spain

${ }^{3}$ La Fe University and Polythecnic Hospital, Valencia, Spain

ABSTRACT: Background: Aripiprazole once-monthly is an LAI formulation of aripiprazole that is currently approved in the USA and Europe for the treatment of schizophrenia. Some studies have reported a decline in hospital admissions and emergency use after initiation on long-acting injectable (LAI) antipsychotics, but the effects of using recently commercialised LAI aripiprazole remains uncertain.

AIMS: To characterize the impact of ALAI initiation on number of hospitalizations and visits to the emergency service, among patients suffering from schizophrenia attending regularly to psychiatric consultations of Gregorio Marañón University General Hospital (Madrid, Spain).

METHODS: Patients initiated on (ALAI) were studied in an observational mirror-image design to assess changes in number of hospitalizations and visits to the emergency service in the 12 months pre- vs 12 months post-depot initiation. Other sociodemographic, physical and clinical variables such as age, gender, weight, blood pressure and presence of dual disorders were also gathered. Variables were collected reviewing clinical records.

Wilcoxon test was used to assess hospitalizations and visits to the emergency. Paired t-tests were used to assess changes in weight and blood pressure. Non parametric Mann-Whitney $U$ test was used to compare aripiprazole doses between genders and in order to assess de influence of dual disorders. In order to perform the statistical analysis, IBM SPSS statistics v.20 was used.

RESULTS: 31 patients were included in the final analysis. Mean age was $44.67(\mathrm{SD}=15.57)$ years. Most of the patients were male $(54.8 \%$ vs $45.2 \%)$. $71 \%$ were previously receiving oral antipsychotics treatment, whereas $29 \%$ were receiving other LAI antipsychotic: no significant differences were observed when comparing hospitalizations $(p=0.74)$ or emergency use $(p=0.98)$ in the 12 months post-initiation 
between these groups. Mean dosage was $352.67 \mathrm{mg} / 28$ days $(\mathrm{SD}=0.461)$, and $38.7 \%$ needed an adjustment during the first year of treatment (dosage increased in 76.9\%). A combination of two or more antipsychotics was prescribed in $64.5 \%$ of the patients. Mean psychiatric number of hospitalizations a year declined from 0.483 /year preinitiation to 0.224 /year post-initiation $(\mathrm{P}<0.05)$, whereas mean visits a year to the emergency psychiatric service declined from 1.419 pre-initiation to 1.032 post-initiation $(\mathrm{P}<0.1)$. No significant changes in weight $(\mathrm{p}=0.82)$, systolic $(p=0.56)$ or diastolic $(p=0.29)$ blood pressure were observed. No gender differences in dosage were observed $(p=0.246)$. Suffering from dual disorders had no influence on dosage either $(p=0.68)$.

CONCLUSIONS: LAI aripiprazole initiation appears to provide a benefit decreasing hospitalization needs and emergency services consumption and it was well tolerated. This data supports previous evidence indicating superiority of LAI antipsychotics.

\section{4}

\section{Long-term Efficacy of Brexpiprazole in Patients with Schizophrenia with Clinically Relevant Levels of Negative Symptoms}

Catherine Weiss, PhD'; Peter Zhang, PhD ${ }^{2}$; Ross A Baker, PhD ; Mary Hobart, PhD ; Nanco Hefting, $\mathrm{MSc}^{5}$; and Stine R Meehan, $P h D^{6}$

${ }^{1}$ Director, Global Medical Affairs, Otsuka Pharmaceutical Development \& Commercialization Inc., Princeton, NJ, USA

${ }^{2}$ Senior Director, Biostatistics, Otsuka Pharmaceutical Development \& Commercialization Inc., Princeton, NJ, USA

${ }^{3}$ Director, CNS Global Medical Affairs, Otsuka Pharmaceutical Development \& Commercialization Inc., Princeton, NJ, USA

${ }^{4}$ Senior Director, Global Medical Affairs, Otsuka Pharmaceutical Development \& Commercialization Inc., Princeton, NJ, USA

${ }^{5}$ Senior Specialist, Clinical Development, H. Lundbeck A/S, Valby, Denmark

${ }^{6}$ Medical Advisor, Medical Affairs Psychiatry,

H. Lundbeck A/S, Valby, Denmark

ABSTRACT: Background: Effective treatments for patients with high levels of negative symptoms of schizophrenia are lacking. Brexpiprazole is a serotonin-dopamine activity modulator that is a partial agonist at 5-HT1A and dopamine D2 receptors, and an antagonist at 5-HT2A and noradrenaline alpha1B/2C receptors, all with subnanomolar potency. Long-term treatment with brexpiprazole demonstrated broad efficacy across all five
Marder factor groupings, including positive, negative, disorganized thoughts, uncontrolled hostility/excitement, and anxiety/depression. This post-hoc analysis of long-term effects of brexpiprazole in patients with clinically relevant levels of negative symptoms of schizophrenia is based on data from two similarly designed short-term, placebo-controlled studies (Vector; NCT01396421 or Beacon; NCT01393613) for the brexpiprazole-treated patients who continued into an open-label extension study (Zenith; NCT01397786).

METHODS: In the short-term studies, patients with acute schizophrenia were randomly assigned to fixed oncedaily doses of brexpiprazole $0.25 \mathrm{mg}$ (Vector), $1 \mathrm{mg}$ (Beacon), $2 \mathrm{mg}, 4 \mathrm{mg}$ or placebo for 6 weeks. The longterm study was an open-label, 52-week (amended to 26 weeks), safety extension study with flexible-dose (1-4 mg/day) brexpiprazole. The post-hoc analyses were performed on brexpiprazole-treated patients from the short-term studies who continued into the long-term study, and who had clinically relevant negative symptoms, defined as PANSS Factor Score for Negative Symptoms (PANSS-FSNS; N1, N2, N3, N4, G7, G16) of $\geq 24$, and score of $\geq 4$ on at least two of three core negative symptom PANSS items at randomization in the parent study. The outcome of the analysis included change from baseline to up to 58 weeks in PANSS-FSNS, PANSS Total, and PSP. Safety was also assessed.

RESULTS: A total of 187 patients with clinically relevant levels of negative symptoms in the parent study rolledover into the open-label extension study and were available for analysis. Eighty-three of these patients remained in the studies for 58 weeks. Due to the study amendment, not all patients had the opportunity of complete 52 weeks of open-label treatment. Baseline PANSS Total score was 104.4, while baseline PANSSFSNS was 27.6 and baseline PSP Total score was 41.3. Mean change (SD) from baseline in PANSS-FSNS was -10.9 (5.0), and -44.2 (17.5) for PANSS Total score at Week 58. Change from baseline (SD) to Week 58 for PSP Total score was 24.8 (12.9) with improvement in all domains (socially useful activities, personal and social relationship, self-care, and disturbing and aggressive behaviors). The TEAEs reported $\geq 5 \%$ were schizophrenia $(18.9 \%)$, insomnia $(8.6 \%)$, weight increased $(5.9 \%)$ and akathisia $(5.9 \%)$.

CONCLUSION: This post-hoc analysis suggests that brexpiprazole has long-term effectiveness on negative symptoms and functioning in patients with schizophrenia and clinically relevant levels of negative symptoms.

Funding Acknowledgements: The study was funded by Otsuka Pharmaceutical Development \& Commercialization Inc. and H. Lundbeck A/S 